\title{
Ideal Time-Based Voltage Control using Evolutionary Algorithm in Distributed Generator Centered Networks
}

\author{
B Vivekanandam, \\ Senior Lecturer, \\ Faculty of Computer Science and Multimedia, \\ Lincoln University College, Malaysia. \\ Email: vivekanandam@lincoln.edu.my
}

\begin{abstract}
The distributed generators (DGs) consists of radial rural distribution networks that makes use of off-voltage tap changing transformers. Ideal tap changer positions for these transformers can be determined using the novel estimation technique proposed in this paper. A branchy low-voltage network is brought down to its equivalent line along with the utilization of spatial network decomposition in this technique. Evolutionary algorithm is used for determining the PV nodes ideal voltage module values in ideal seasonal control plan. A PQ node with 3 DGs incorporated in a radial 40-node network and a PQ node with 10 DGs are incorporated in a radial 33-node network are the distribution networks used for modelling the proposed system.
\end{abstract}

Keywords: Evolutionary Algorithm, distributed generator (DG), voltage control, distribution network, off-voltage tap changing transformer;

\section{Introduction}

In distributed networks, the voltage conditions and power flow is influenced significantly by distributed generators [1]. In distribution networks that consists of DGs, voltage control is a significant power function. Offvoltage tap changing transformers (OVTCT) and under-load tap changing transformers (ULTCT) are the basic voltage control resources available in the distribution networks [2]. The influence of DGs on voltage control resources are examined and ideal voltage control is achieved by suggesting several measures essential for mutual coordination. In most cases, ULTCT is considered. In rural distribution networks, OVTCT is the only available control resource [3]. Over any specified time period, we regard voltage control as a segment in the exploitation strategy of the ideal distribution network. The OVTCT ideal tap changer position and settings are to be determined to solve this issue. Minimum power loss and voltage deviation must be achieved in the distribution network while aiming for control based on the DGs and predicted time duration based power diagram. Type, location and power of the DGs impact the solution and course of solving this issue. The operation mode and connection type largely affects the ability of participation of the DG in the voltage control process [4].

The possible typical cases include - providing uniform active and reactive supply load and output power from the DG to a large load located near it [5]. At the connection point, the total power load is reduced to bring down the impact of DG in this scenario. Modelling the DG as a negative power load can be performed. AB node is the node where DG is connected. A nearly constant power factor is used for injecting the active power by the DG. As the DG's active power increases, the voltage at the connection point of the DG also increases. Modelling the DG as a negative power load can be done. AB node is the node where DG is connected in this scenario also [6]. At the connection point, power is injected by the DG with specific voltage. The voltage is controlled by controlling the injection of reactive power at the connection point by the DG. PV node is the node at which such DG is attached. They offer significant participation in the distribution network voltage control [7]. In rural distribution networks consisting of DGs, the ideal voltage control plan for a specified time duration can be determined using the scheme presented in this paper. The voltage control problem and spatial decomposition principle forms the basis of this procedure.

\section{Time Specific Ideal Voltage Control}

A simple rural radial distribution network is represented in figure 1. It consists of Low voltage feeders or networks, several medium or low voltage transformers, medium voltage network, medium voltage supply transformed and a medium voltage network feed point node. AB nodes are the DGs and the consumers. Offvoltage tap changing transformers are used for all medium voltage and medium or low voltage transformers [3]. 
The low voltage networks, medium and low voltage transformers and medium voltage networks are covered by the medium voltage transformers and their voltage control activity. The medium and low voltage transformers supply to the low voltage network and they are not influenced by the medium and low voltage transformer control activities owing to the networks radial structure. The voltage control and its spatial decomposition is performed on this basis [8]. The time based ideal voltage control problem can be addressed in two levels. The medium and low voltage transformers ideal tap changer positions are determined at the first level. The medium voltage power supply transformers ideal tap changer position is determined in the second level.

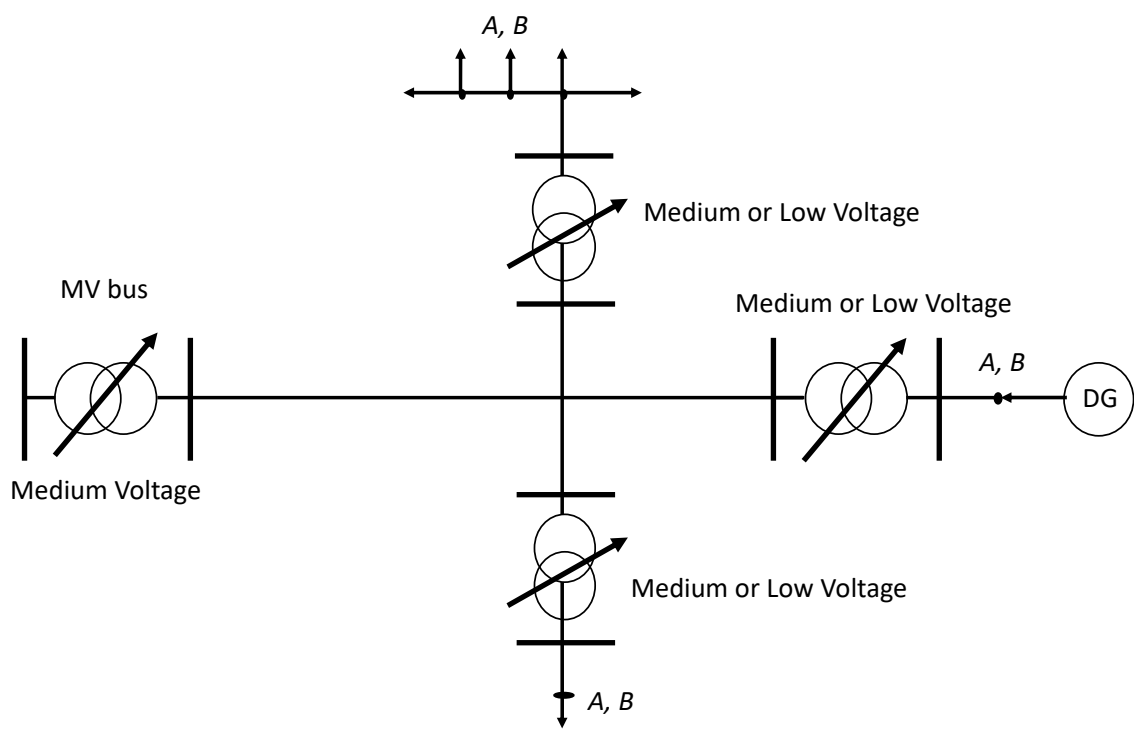

Figure 1: Rural distribution network architecture

At the first level, at each medium or low voltage distribution transformer, the ideal tap changer position is determined independently. With respect to the transformer tap changer position, the voltage of medium or low voltage transformers medium voltage bus is maintained constant for any specific power of the low voltage consumer [9]. The desired voltage on the transformer low voltage side, the voltage on the transformer medium voltage bus, and the power consumption on the transformer low voltage side are used for determining the ideal tap changer position. Optimization criterion is the issue in selection of desired value for voltage [10]. Nominal voltage values are used for implicitly determining the system ideal operation. Maintaining nominal consumer voltage values to the maximum level is the major goal of time-based voltage control [11].

\section{Proposed Work}

The time-based ideal voltage control issue is solved by regulating the medium voltage supply transformers tap changer position [12]. While overcoming this issue, the optimization criteria has to be satisfied along with the voltage limitation criteria. Damage minimization caused by deviation of voltage from the nominal value, minimization of voltage sag and minimization of loss can be the mentioned criteria. For all the tap changer positions of the supply transformer, the power flow calculation is performed to execute the optimization task. The medium or low voltage transformer current tap changer position is determined during each calculation of power flow [13]. Further, the objective function value is also estimated parallelly. In any specific distribution network, the time based ideal control plan for distribution transformers with minimum objective function value represents the ideal solution. The control plan is determined by the optimization criterion that is adopted. Figure 2 represents the flowchart of the proposed model. 


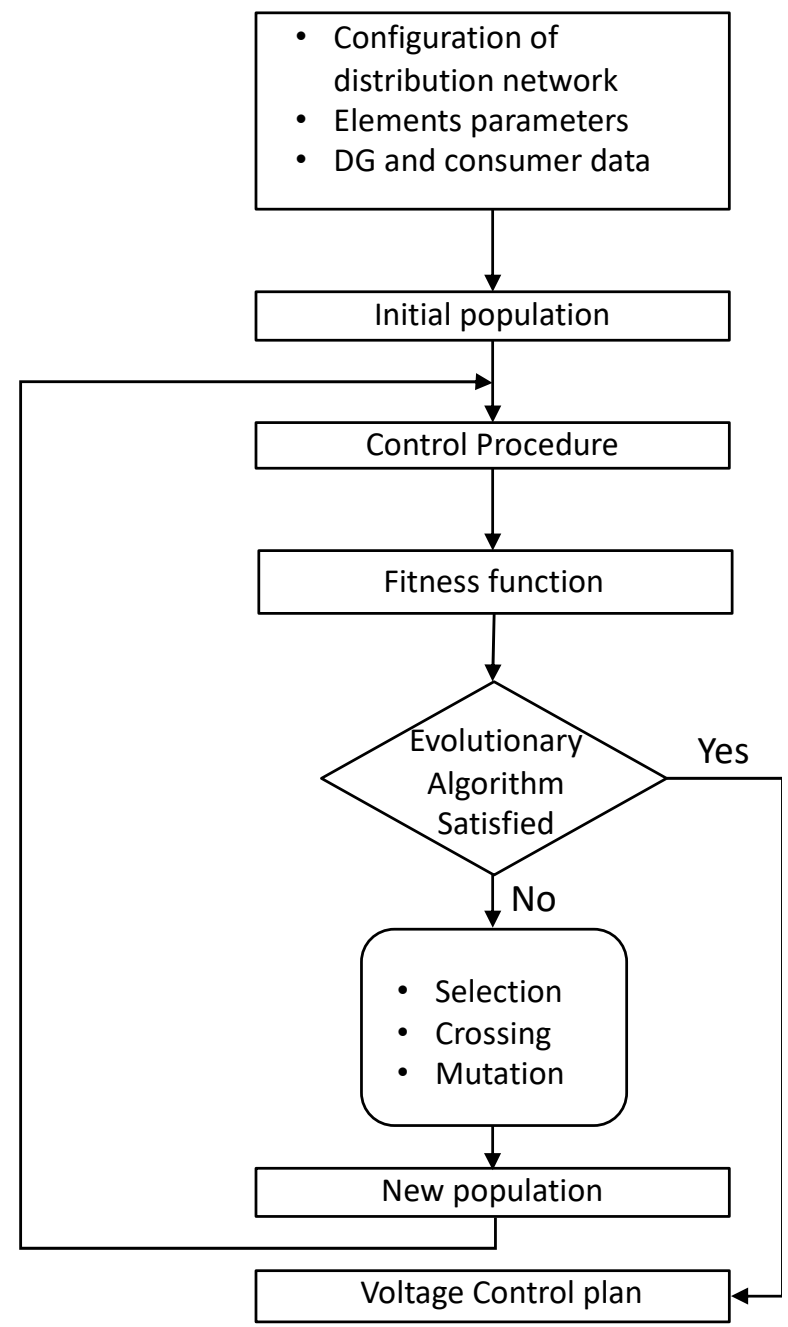

Figure 2: Flowchart for time-based voltage control using Evolutionary Algorithm

Constant voltage and active power module is maintained for operation of the PV node modelled by the DG. The DG or PV node reactive power injection is changed for compensating the influence of distribution network power change on the voltage of the PV node. The voltage of PV node is maintained at specific value by this technique [14]. Independent selection or choice of PV nodes voltage module values under a certain range can be performed. For specified conditions of the distribution network, the possible alteration range of the DG reactive power and active power range, the mentioned range can be determined. It is essential to determine the PV node values of the ideal voltage modules in the time-based ideal seasonal voltage control plan [15]. The time-based ideal control plan can be determined by the application of evolutionary algorithm as represented in figure 2 . Based on the provided objective function, the evolutionary algorithm selects multiple combinations of voltage values for the PV nodes. MATLAB tool is used for implementing the presented evolutionary algorithm.

\section{Results and Discussion}

Two distribution networks are used for testing and analysis of the proposed system. The DGs annual power diagrams are analyzed along with the voltage profile of the distribution network. Figure 3 represents voltage ratio correction at the transformer 10 and 15 with time based ideal voltage control plan. There is a decrease in voltage at node 13 and 20. It is brought within the standard limits to a range of 0.9 to 1 per unit. The voltage at node 12 is improved by the time-based ideal voltage control if minimal simultaneous power is provided by the DG. However, when compared to the minimum allowed standard, the value is still lower. The time-based voltage controls limited domain can be implicated by improving the voltage profile to the maximum level at given circumstances as represented by figure 4 . The DGs and consumers time-based load diagrams and network data is obtained. Equivalent lines are used for substituting the low voltage networks. The medium voltage transformer tap changer position is set at the minimum value and the power flow calculation is performed. 
Pages: 233-238

https://www.irojournals.com/iroei/

DOI: https://doi.org/10.36548/jei.2020.4.006

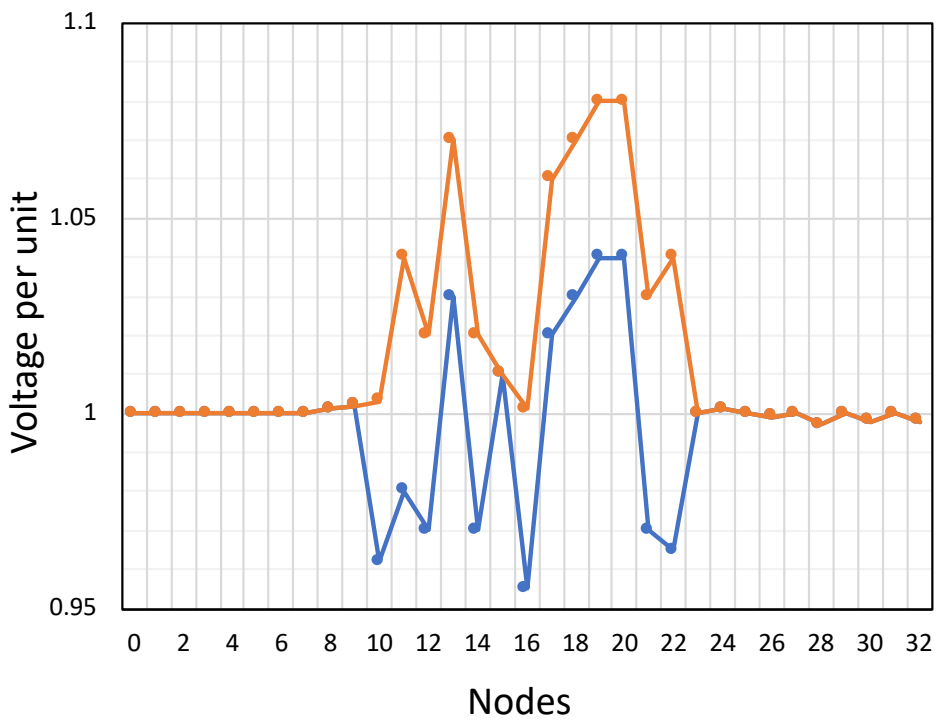

Figure 3: Maximal network voltage profile of the micro hydro power plant

Form the DG power diagrams, two typical periods may be identified. The lower power period ranges between July to December and the higher power period ranges between January to June. Hence two seasons are considered for applying the proposed procedure. The micro hydro power plant mean values are also considered simultaneously for these two periods. Slight power variations and low power consumption are common in rural area. For this reason, the constant power consumption value is considered.

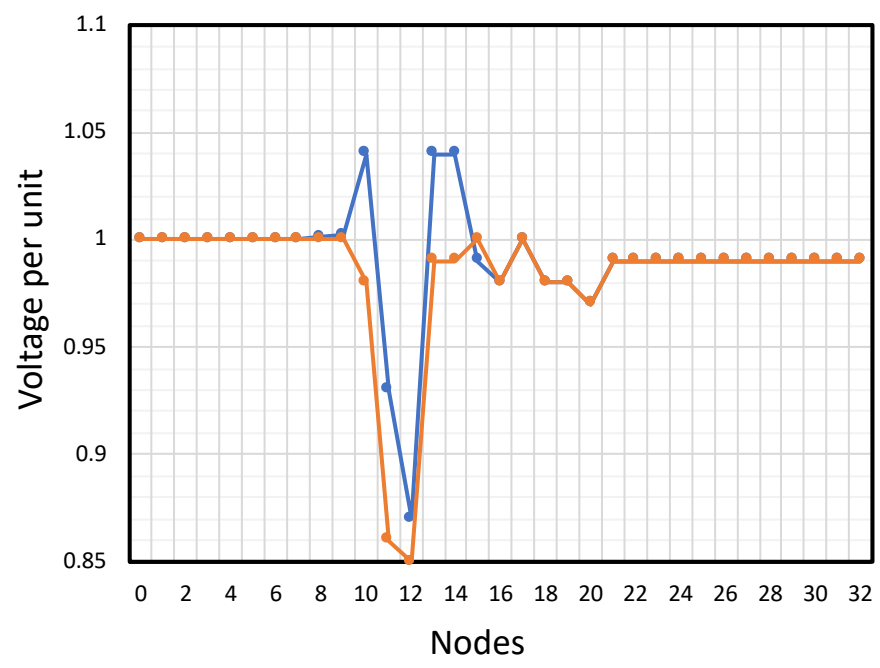

Figure 4: Minimal network voltage profile of the micro hydro power plant

The nominal consumer power and network branch impedance data is taken into consideration during calculation. Figure 5 represents the normalized power diagram for the duration of one year. The DG characteristics are also taken into consideration. Two selected nodes are connected to the voltage modules. The reactive power maybe controlled by the DGs that deliver constant active power. Constant active power consisting of a fixed power factor is injected by the DG linked with node 20 . Figure 5 provides the mean power values determined for the time-based ideal voltage control scheme. A significant portion of the time-based ideal voltage control plan is represented by the PV nodes ideal voltage values other than the transformers ideal tap changer positions. 


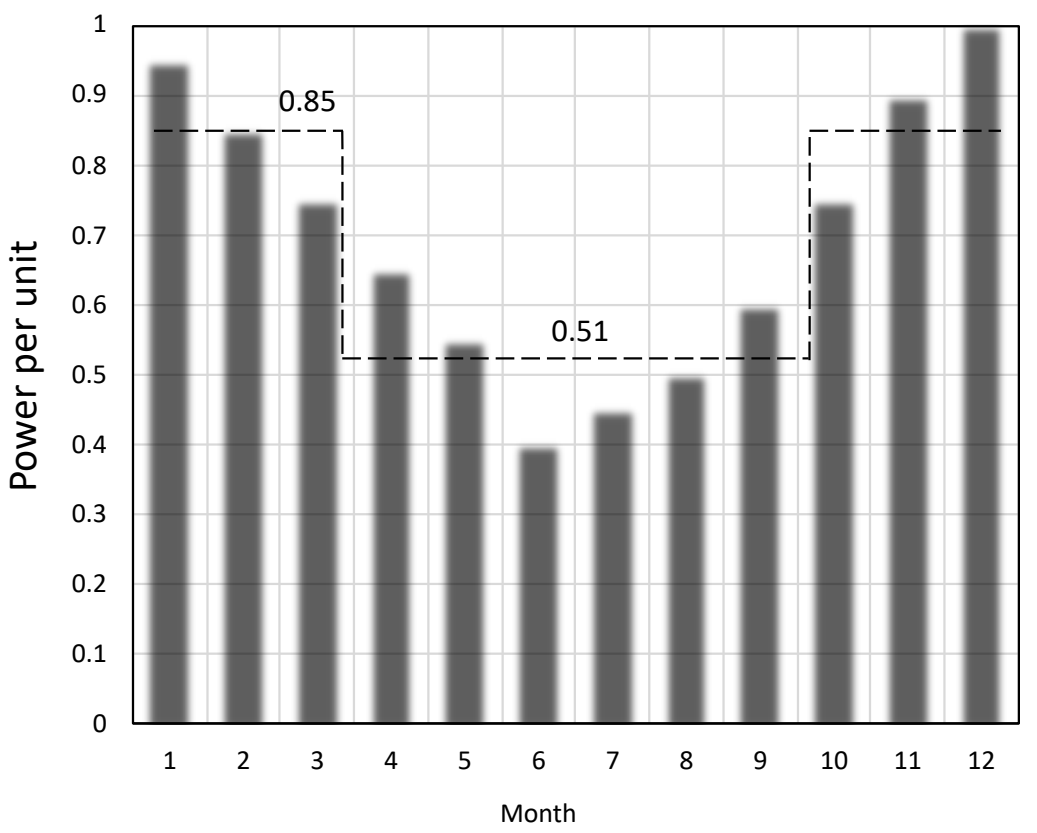

Figure 5: Normalized power diagram for one year duration

\section{Conclusion}

For rural distribution network consisting of distributed generators, an time-based ideal voltage control plan is presented and tested in this paper. PV node voltage correction and network voltage profile improvement applying evolution algorithm, assigning the medium or low voltage distribution transformed with ideal transfer ratio to bring the low voltage network down to its equivalent line, and the voltage control issues spatial decomposition principle are the significant characteristics of this technique. AB and PV nodes are modelled with huge quantity of DFs in the distribution network to test the practical applications of the proposed scheme. Voltage variation occurs beyond the standard limits due to the micro hydro power plant space dispersion and significant generated power variations. With an exception of certain outlying nodes, the voltage value is maintained within a standard limit by the transformers time-based voltage control under ideal mode. Multiple node distribution networks are modelled with DGs as PV and AB nodes which has proven significant improvement in ideal voltage correction and network voltage profile. Future work is directed towards increasing the number of nodes for timebased voltage control at the transformers and real time analysis of power distribution.

\section{References}

[1] Momesso, A. E., Bernardes, W. M. S., \& Asada, E. N. (2019). Fuzzy adaptive setting for time-currentvoltage based overcurrent relays in distribution systems. International Journal of Electrical Power \& Energy Systems, 108, 135-144.

[2] Emmanuel, N. (2018). Design of a Fuzzy Logic Based adaptive protection scheme in distribution Networks with Distributed Generator (Doctoral dissertation, Emmanuel Nsengiyumva).

[3] Veloso, C. G., Rauma, K., FernándezOrjuela, J., \& Rehtanz, C. (2020). Real-time agent-based control of plug-in electric vehicles for voltage and thermal management of LV networks: formulation and HIL validation. IET Generation, Transmission \& Distribution, 14(11), 2169-2180.

[4] Wang, Y., Wang, Y., Huang, Y., Yang, J., Ma, Y., Yu, H., ... \& Zhang, Y. (2019). Operation optimization of regional integrated energy system based on the modeling of electricity-thermal-natural gas network. Applied Energy, 251, 113410.

[5] Ponnaganti, P., Pillai, J. R., \& Bak-Jensen, B. (2018). Opportunities and challenges of demand response in active distribution networks. Wiley Interdisciplinary Reviews: Energy and Environment, 7(1), e271.

[6] Ishii, H., \& Hayashi, Y. (2017, December). Optimal smart functions of large-scale PV inverters in distribution systems. In 2017 IEEE Innovative Smart Grid Technologies-Asia (ISGT-Asia) (pp. 1-7). IEEE.

[7] Li, R., Wang, W., Chen, Z., Jiang, J., \& Zhang, W. (2017). A review of optimal planning active distribution system: Models, methods, and future researches. Energies, 10(11), 1715. 
[8] Canha, L. N., Pereira, P. R., Milbradt, R., da Rosa Abaide, A., Schmitt, K. E. K., \& de Abreu Antunes, M. (2017, August). Intelligent voltage regulator to distributed voltage control in smart grids. In 2017 52nd International Universities Power Engineering Conference (UPEC) (pp. 1-6). IEEE.

[9] Dao, V. T., Ishii, H., \& Hayashi, Y. (2019). Optimal parameters of volt-var functions for photovoltaic smart inverters in distribution networks. IEEJ Transactions on Electrical and Electronic Engineering, 14(1), 75-84.

[10] Rabiee, A., Mohseni-Bonab, S. M., Parniani, M., \& Kamwa, I. (2018). Optimal cost of voltage security control using voltage dependent load models in presence of demand response. IEEE Transactions on Smart Grid, 10(3), 2383-2395.

[11] Jafari, M., Olowu, T. O., \& Sarwat, A. I. (2018, September). Optimal smart inverters volt-var curve selection with a multi-objective volt-var optimization using evolutionary algorithm approach. In 2018 North American Power Symposium (NAPS) (pp. 1-6). IEEE.

[12] Antoniadou-Plytaria, K. E., Kouveliotis-Lysikatos, I. N., Georgilakis, P. S., \& Hatziargyriou, N. D. (2017). Distributed and decentralized voltage control of smart distribution networks: Models, methods, and future research. IEEE Transactions on smart grid, 8(6), 2999-3008.

[13] Kumar, D. (2019). Review on task scheduling in ubiquitous clouds. Journal of ISMAC, 1(01), 72-80.

[14] Wang, H. Flexibility Management in Renewable Energy Source Operated Power Systems using Decision Support System.

[15] Nirmal, D. (2020). Artificial Intelligence Based Distribution System Management and Control. Journal of Electronics, 2(02), 137-47. 